\title{
A SIMPLE MONOCHROMATOR FOR ULTRAVIOLET MICROSCOPY
}

\author{
M. T. JANSE N \\ Department of Histology, Faculty of Medicine, University of Utrecht, The Netherlands
}

Received August 10, 1958

$I_{N}$ most commercial monochromators the aperture of the exit beam is much larger than that of a microscope condenser in its object space. Accordingly, unless extra mirrors or lenses are used, a considerable amount of light is lost and the dispersive power of the instrument is not fully utilized (e.g. $[1,2]$ ).

We have found that a very useful and extremely simple monochromator can be built with an aperture ratio of the exit beam of $1: 70$ if the parts are arranged as in Fig. 1. There is one concave spherical mirror $S_{1}$ which yields an image of the entrance slit in the plane of the exit slit. Of the rays passing through the prism those nearest to the refracting rib are made to be minimally refracted. As the beam is converging, the other rays will undergo an ever greater than minimal deviation according as their distance from the refracting rib increases. It has been found that this results in a remarkably good compensation of the image faults caused by the off-axis position of the spherical mirror. For the purpose in hand the effectiveness of this compensation is amply sufficient throughout the visible and ultraviolet spectra as is evidenced by the high pressure mercury arc line images in Figs. 2 and 3 . The complete reversal of the mercury line $254 \mathrm{~m} \mu$ that occurs in the arc we used may serve to give an estimate of the amount of stray light present in the ultraviolet part of the spectrum (see Fig. 3). The relative intensities of the spectrum have been measured by means of a 1 P $28 \mathrm{RCA}$ photomultiplier tube. It was found that at the $254 \mathrm{~m} \mu$ setting of the monochromator, the photomultiplier current was less than 1 per cent of that at either 248 or $257 \mathrm{~m} \mu$. Apparently, the amount of stray light in the monochromator is negligible when used with a mercury arc.

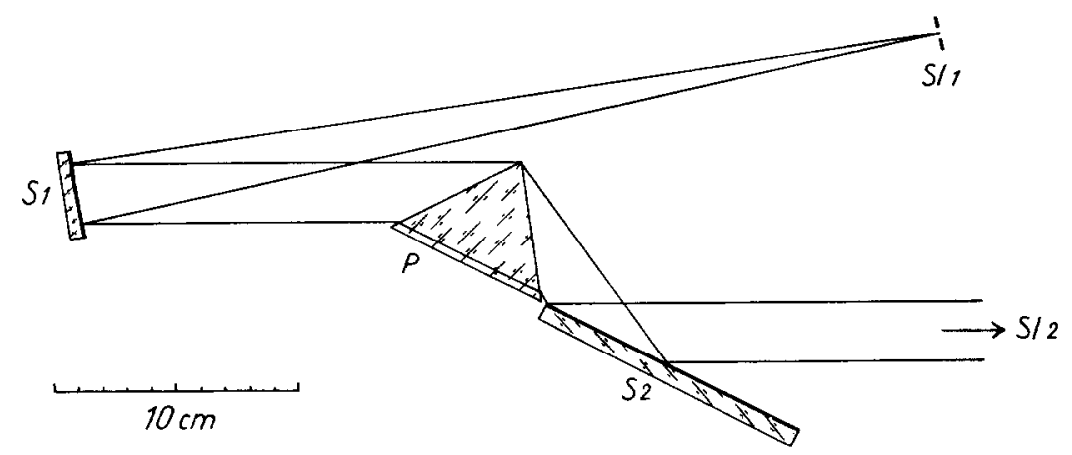

Fig. 1.- Diagram of the monochromator. The greater part of the exit beam and the exit slit have been omitted. $\mathrm{Sl}_{1}$, entrance slit; $\mathrm{S}_{1}$, concave spherical mirror; P, prism; $\mathrm{S}_{2}$, flat mirror, $\rightarrow \mathrm{Sl}_{2}$, towards exit slit at $200 \mathrm{~cm}$ from $S_{1}$. 


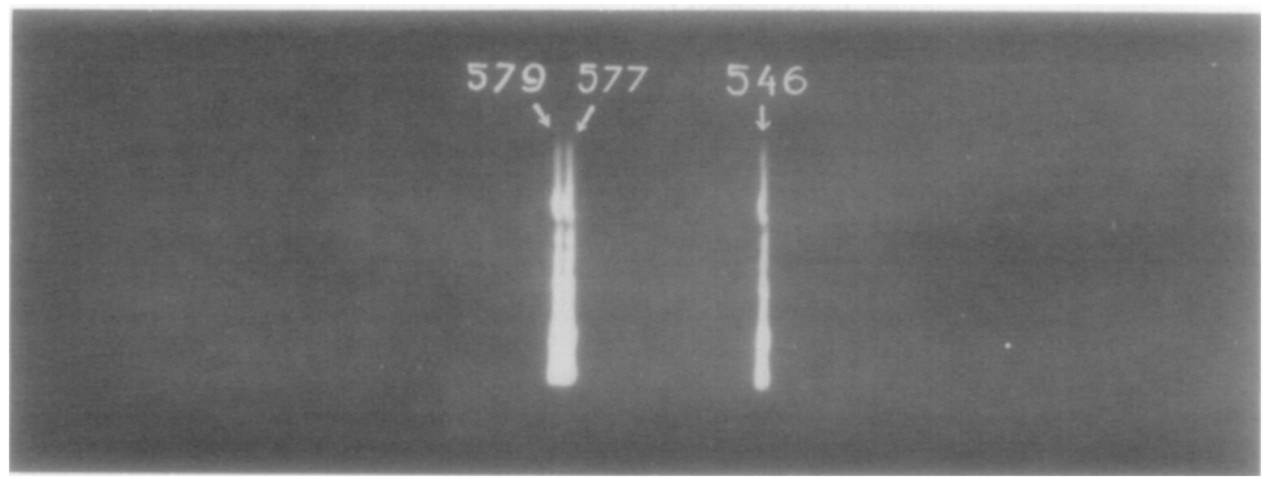

Fig. 2.-Part of the visible spectre photographed by substituting a platc for the exit slit of the monochromator. Numbers denote wavelenghts in $\mathrm{m} \mu$.

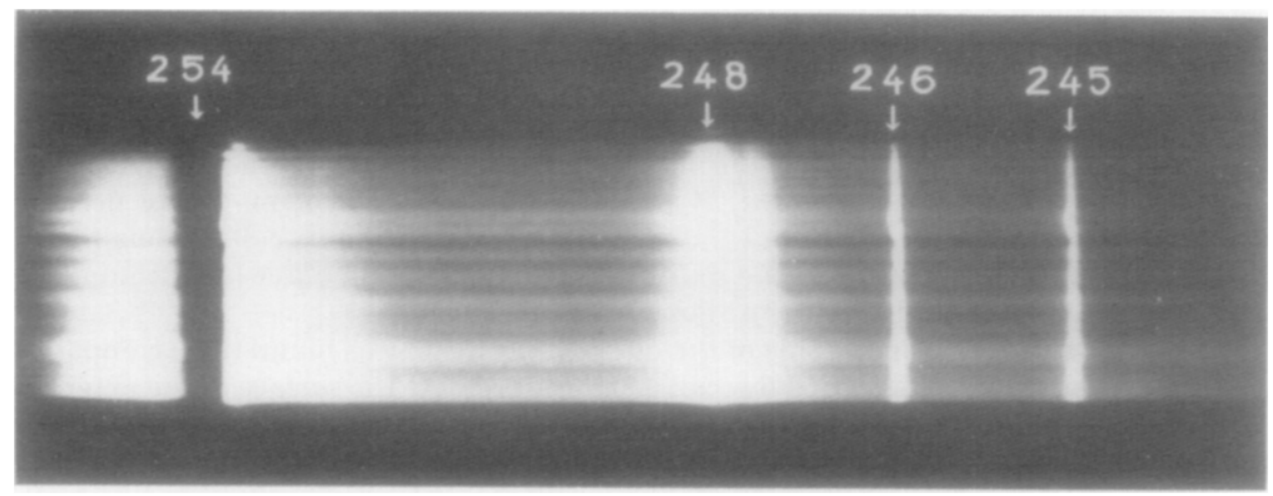

Fig. 3.-Part of the ultraviolet spectre. Though heavily overexposed the reversed line at $254 \mathrm{~m} \mu$ is completely dark.

The skillful assistance of Mr. W. Kerssen, who built the mechanical parts of the monochromator, is gratefully acknowledged.

\section{REFERENCES}

1. Loofbourow, J. R., J. Opt. Soc. Am. 40, 317 (1950).

2. Walker, P. M. B., Physical techniques in biological research. Vol. III, p. 401. Academic Press Inc., New York, 1956. 